\title{
Análise espacial da qualidade dos serviços de Atenção Primária à Saúde na redução da mortalidade infantil
}

\author{
Spatial analysis of the quality of Primary Health Care services \\ in reducing child mortality
}

Amanda Namíbia Pereira Pasklan (https://orcid.org/0000-0001-7193-4861) ${ }^{1}$ Rejane Christine de Sousa Queiroz (https://orcid.org/0000-0003-4019-2011) ${ }^{2}$ Thiago Augusto Hernandes Rocha (https://orcid.org/0000-0002-6262-3276) ${ }^{3}$ Núbia Cristina da Silva (https://orcid.org/0000-0002-0809-2152) ${ }^{3}$

Aline Sampieri Tonello (https://orcid.org/0000-0002-8402-9112) ${ }^{2}$

João Ricardo Nickening Vissoci (https://orcid.org/0000-0001-7276-0402) ${ }^{4}$

Elaine Tomasi (https://orcid.org/0000-0001-7328-6044) ${ }^{5}$

Elaine Thumé (https://orcid.org/0000-0002-1169-8884) ${ }^{5}$

Catherine Staton (https://orcid.org/0000-0002-6468-2894) ${ }^{4}$

Erika Bárbara Abreu Fonseca Thomaz (https://orcid.org/0000-0003-4156-4067) ${ }^{2}$
${ }^{1}$ Programa de Pós-

Graduação em Saúde Coletiva, Universidade Federal do Maranhão (UFMA). R. Barão de Itapary 155, Centro. 65020070 São Luís MA Brasil. amanda_namibia@hotmail. com

${ }^{2}$ Departamento de Saúde Pública, UFMA. São Luís MA Brasil.

${ }^{3}$ Centro de Pós-Graduação e Pesquisa em Administração, Universidade Federal de Minas Gerais. Belo Horizonte MG Brasil.

${ }^{4}$ Duke University. Durham NC USA.

${ }^{5}$ Universidade Federal de Pelotas. Pelotas RS Brasil.

\begin{abstract}
This study sought to analyze the correlation of the quality of Primary Health Care services in reducing child mortality, via geoprocessing. It involved an ecological study, with a crosssectional approach, in which secondary data from all 5,565 Brazilian municipalities were used to analyze the infant mortality rate (IMR) and cause of infant death. The data related to IMR was obtained from the Mortality Information System. For the spatial analysis, 5,011 municipalities were included. The clustering analyses were performed using GEODA software and the spatial regression analyses were performed using ARCGIS 10.5 software. In Brazil, there was a $45.07 \%$ reduction in IMR between the years 2000 and 2015. The greatest reduction occurred in the northeastern region of the country, although it is still the region with the highest IMR. Of the 749 municipalities analyzed in the differential cluster for infant death, 153 had high IMR. The areas with the greatest increase in IMR were found in the North and Northeast regions. In Brazil, IMR proved to be inversely associated with the accessibility to high complexity services, health management strata and population size, reference for childbirth, live birth rate, per capita income and unemployment rate. A progressive reduction in IMR was recorded between 2000 and 2015.
\end{abstract}

Key words Epidemiology, Infant Mortality, Primary Health Care
Resumo Objetivou-se analisar a correlação da qualidade dos serviços da Atenção Primária na redução da mortalidade infantil, através do geoprocessamento. Um estudo ecológico, de abordagem transversal, em que foram utilizados dados secundários de todos os 5.565 municípios brasileiros para análise da taxa de mortalidade infantil (TMI) e causa de óbito infantil. Os dados da TMI foram obtidos no Sistema de Informação de Mortalidade. Para a análise espacial, incluímos 5.011 municípios. As análises de clusterização ocorreram no software GEODA e as análises de regressão espacial no ARCGIS 10.5. No Brasil houve uma redução de 45,07\% da TMI entre os anos $2000 e$ 2015. A maior redução ocorreu na região nordeste do país, apesar de ainda ser a região com maior número na TMI. Dos 749 municípios analisados no cluster diferencial para óbito infantil, 153 apresentaram alta TMI. As áreas com maior expansão de alta TMI foram encontradas nas regiões Norte e Nordeste. No Brasil, a TMI mostrou-se inversamente associada à acessibilidade aos serviços de alta complexidade, ao estrato da gestão em saúde e porte populacional, à referência para o parto, à taxa de nascidos vivos, à renda per capita e à taxa de desemprego. Verificou-se uma crescente redução da TMI entre o período de 2000 a 2015. Palavras-chave Epidemiologia, Mortalidade Infantil, Atenção Primária à Saúde 


\section{Introdução}

Em 1978, foi realizada uma conferência internacional, em Alma Ata, com a proposta de ampliar a qualidade e o papel da atenção primária a saúde (APS), de forma que garantisse o princípio da justiça social e o acesso universal aos serviços de saúde ${ }^{1,2}$. Nessa conferência, foram discutidas metas para o alcance da saúde até o ano 2000. A resolução aprovada reconheceu os cuidados de saúde primários como um direito universal. Nessa lógica, investimentos na APS e a organização sistemática do processo de trabalho nos serviços de saúde foram realizados ${ }^{1,3}$.

Estudos demonstram que grandes conquistas foram alcançadas na APS, como: maior equidade no acesso aos cuidados de saúde, redução de vulnerabilidades a riscos sociais e ambientais, melhora dos mecanismos e oportunidades participativos, aumento de políticas intersetoriais sobre os condicionantes sociais e econômicos da saúde, e a redução do óbito infantil, ou seja, a morte de crianças menores de um ano de idade ${ }^{4,5}$.

Para a viabilidade dessa conquista, realizouse um uso mais racional dos recursos ${ }^{1}$, priorizando metas como o aumento do acesso ao serviço de saúde, do planejamento familiar, da cobertura da imunização, e da assistência pré-natal ${ }^{5}$. Tais propostas surgiram a partir dos atributos propostos para a APS, um serviço focado na acessibilidade do paciente, família e comunidade, e uma atenção integral e coordenada, orientada para o paciente ao longo do tempo, com interação entre o profissional e o paciente . $^{6}$

A meta da redução do óbito infantil permaneceu enfatizada no Relatório Mundial de Saúde da Organização Mundial de Saúde (OMS) nos objetivos de desenvolvimento pós-2015. $\mathrm{Na}$ agenda 2030 para o Desenvolvimento Sustentável, metas de saúde da criança foram abordadas com o objetivo de assegurar às crianças menores de um ano uma vida saudável, com promoção do bem-estar ${ }^{7}$.

Dessa forma, completados 40 anos da conferência, ainda existem desafios a serem superados, principalmente nos países pobres e em países em desenvolvimento. A persistência de problemas discutidos antes mesmo da Alma-Ata é preocupante, como o grande número de óbitos infantis ${ }^{3}$.

Sabe-se que ainda existem desafios a serem superados para o alcance pleno da proposta da declaração de Alma Ata, inclusive em relação à mortalidade infantil (MI). No Brasil, a MI ainda é um importante problema, especialmente nos segmentos mais pobres da população, visto que aspectos socioeconômicos e demográficos também estão relacionados ${ }^{3,8-10}$.

Sabe-se que estudos demonstram que a APS tem um papel central para a efetivação dessa conquista $^{11-16}$. Ainda são poucas as abordagens científicas baseadas em geografia da saúde para avaliar desfechos de políticas em saúde na redução da MI no Brasil. Dessa forma, propõe-se aqui analisar a correlação da qualidade dos serviços da APS na redução da MI, através do método do geoprocessamento, e discutir as dificuldades ainda presentes no Brasil com relação ao alcance das metas internacionais de redução da MI.

\section{Método}

Trata-se de um estudo ecológico, de abordagem transversal, em que a coleta dos dados ocorreu no período de julho 2016 a maio de 2018. Para análise do comportamento da TMI ao longo dos anos 2000 a 2015, foram confeccionados gráficos no Programa Excel-Microsoft. O gráfico de linha foi utilizado para detalhar a tendência da MI por região, o gráfico de coluna para o detalhamento por estado brasileiro, e o gráfico de área para análise das causas de morte infantil. Criou-se também neste programa uma lista com o ranking de MI dos estados brasileiros, comparando a posição de cada unidade federativa (UF) entre os anos 2000 e 2015.

Para analisar os padrões de distribuição e agrupamento da taxa de MI no contexto brasileiro, foram utilizados dados secundários referentes às informações sobre a MI; confundidores econômicos e demográficos e demandas de serviços no Brasil; estrutura e processo de trabalho na APS; índice de acessibilidade a serviços de alta complexidade; estratificação de municípios brasileiros para fins de avaliação de desempenho em saúde.

Os dados relacionados à TMI foram obtidos junto ao Sistema de Informação de Mortalidade (SIM) disponíveis no Departamento de Informática do Sistema Único de Saúde (DATASUS) ${ }^{17}$, compreendendo o período de 2000 a 2015. Dados sobre os confundidores econômicos e demográficos e demandas de serviço foram obtidos junto ao Instituto Brasileiro de Geografia e Estatística $(\text { IBGE })^{18}$ e DATASUS.

Foram consideradas as seguintes variáveis: proporção de menores de cinco anos, taxa de nascidos vivos, taxa de analfabetismo, renda per capita, taxa de desemprego, proporção de parto vaginal, e proporção de parto hospitalar. 
Os dados referentes à estrutura e processo de trabalho na APS foram obtidos do Programa de Melhoria do Acesso e da Qualidade da Atenção Básica (PMAQ-AB) ${ }^{19}$, relacionados ao segundo ciclo de monitoramento do programa, ocorrido em 2014. Foram selecionadas todas as equipes de atenção básica $(\mathrm{AB})$ participantes do programa $(n=30.522)$, em 5.077 municípios brasileiros. Perdas por incompletude dos dados levaram a uma amostra final de 29.778 equipes de APS, e 24.055 unidades básicas de saúde (UBS), distribuídos em 5.011 municípios.

As variáveis selecionadas foram: equipe mínima, equipamentos e materiais, insumos, imunobiológicos, testes diagnósticos, horário mínimo de funcionamento (funcionar pelo menos cinco dias na semana), funcionar em dois turnos, visita domiciliar, educação permanente, coordenação do cuidado, referência para o parto, consulta no pré-natal, consulta de puericultura, promoção da saúde, e planejamento familiar.

As variáveis referentes à equipe mínima, equipamentos e materiais, insumos, imunobiológicos, e testes diagnósticos foram agrupadas segundo a média em um escore, denominado infraestrutura, que serviu de proxy como os parâmetros de estrutura das UBS, nas quais as equipes de saúde estavam alocadas.

As variáveis de horário mínimo de funcionamento, funcionar em dois turnos, visita domiciliar, educação permanente, coordenação do cuidado foram agrupadas de forma semelhante em um escore, denominado disponibilidade, para evidenciar o status da disponibilidade da rede de cuidado.

As variáveis referentes à promoção da saúde e planejamento familiar foram agregadas em um escore relacionado às ações de promoção da saúde e planejamento familiar. As demais variáveis (referência para o parto, consulta no pré-natal e consulta de puericultura) foram mantidas nas análises sem agrupamento.

$O$ índice de acessibilidade à rede de emergência foi obtido junto ao trabalho desenvolvido por Rocha et al. ${ }^{20}$ que analisou a acessibilidade a serviços de alta complexidade em todo o território brasileiro, utilizando metodologias de geoprocessamento. Os dados referentes à classificação dos municípios brasileiros para fins de avaliação de desempenho de serviços de saúde foram obtidos junto ao trabalho de Calvo et al. ${ }^{21}$. Essa classificação permite agrupar os municípios brasileiros segundo uma série de fatores determinantes para a avaliação de políticas em saúde, com foco em desempenho. Assim, é possível estratificar os mu- nicípios do país segundo parâmetros de similitude, e tecer comparações entre municípios com características mais próximas.

Para analisar associações entre os diferentes aspectos da oferta de serviços de APS e determinantes sociais com as tendências temporais de MI no país, foram utilizadas três abordagens baseadas em geografia da saúde. Primeiramente, foi realizada uma análise de clusterização espacial, baseada na abordagem diferencial da estatística local de I de Moran ${ }^{22}$.

A partir do uso dessa técnica, foi possível analisar de forma diferencial a presença de clusters espaciais referentes às TMI nos municípios brasileiros no período de 2000 a 2015. Essa análise aponta a presença de clusters de valores High- $\mathrm{Hi}$ gh, Low-Low e os outliers correspondentes aos clusters Low-High e High-Low. Os seus resultados permitem evidenciar mudanças ao longo do tempo, quanto ao fenômeno observado.

Considerando a distribuição dos clusters, seria desejável traçar estratégias diferenciadas para identificar quais foram as variáveis que se associaram à MI e se a equação global estabelecida para o país era não estacionária. Para tanto, foi realizada uma segunda etapa referente a uma análise exploratória de múltiplas combinações de regressões espaciais com abordagem de mínimos quadrados ordinários (MQO) $)^{23,24}$.

Uma vez que foram apresentados indícios (estatística de Koenker (BP) significante) de que a MI era não estacionária, foi realizada uma análise de regressão geograficamente ponderada (Geographically Weighted Regression - GWR), para a definição de equações customizadas para cada município ${ }^{25}$.

Essa estatística, quando significativa, aponta que o fenômeno observado é não estacionário. Um fenômeno geográfico não estacionário é aquele que tem a sua explicação variando de forma não uniforme ao longo do território analisado. Isso significa que para uma análise de regressão os coeficientes das variáveis preditoras apresentam pesos diferenciados para as diferentes localidades, sendo assim o fenômeno analisado pode ser melhor examinado com o uso da técnica de GWR.

Para fins da análise exploratória baseada em MQO, foi considerada como medida das variáveis independentes e desfecho da TMI ajustada por população, a média no período de 2000 e 2015. Optou-se pelo uso da média após a realização de vários testes com as medidas de desfecho correspondendo a cada ano de forma individual. Os testes levaram à conclusão de que a obser- 
vação pontual referente aos anos não refletia as variações observadas ao longo do período considerado, uma vez que muitos municípios apresentavam dados zerados o que não é bem tolerado pela análise GWR.

A análise exploratória baseada em MQO é feita através de múltiplas interações considerando um grupo de variáveis preditoras, e selecionando o melhor modelo com base em parâmetros como a estatística AIC (Akaike Information Criterion) ou o valor de R2 (Coeficiente de Determinação).

Para fins de análise, o grupo de preditores testados compreendeu as seguintes variáveis disponíveis no estudo: acessibilidade a serviços de alta complexidade, estratificação de municípios brasileiros, infraestrutura, disponibilidade, referência para o parto, consulta no pré-natal, consulta de puericultura, promoção da saúde e planejamento familiar, proporção de menores de 5 anos, taxa de nascidos vivos, taxa de analfabetismo, renda per capita, taxa de desemprego, proporção de parto vaginal, e proporção de parto hospitalar.

A escolha do melhor modelo priorizou aqueles com menor estatística de AIC e com maior R2. A partir da análise exploratória, apenas a variável proporção de parto vaginal não foi selecionada para o exame do modelo espacial baseado em MQO.

A análise de MQO indicou o grupo de variáveis estatisticamente significativas sob uma perspectiva global e as mesmas foram selecionadas para o modelo GWR (acessibilidade a serviços de alta complexidade, estratificação de municípios brasileiros, infraestrutura, referência para o parto, taxa de nascidos vivos, renda per capita, e taxa de desemprego). A análise se mostrou adequada em função da análise de significância da estatística de Koenker junto ao modelo MQO.

As análises de clusterização foram realizadas no software GEODA ${ }^{26}$ e as análises de regressão espacial foram realizadas no ARCGIS 10.5.

\section{Resultados}

Este estudo incluiu dados secundários de todos os 5.565 municípios brasileiros, segundo regiões e estados brasileiros, para análise da TMI e causa de óbito infantil. Dados referentes à estrutura das UBS e processo de trabalho das equipes da atenção básica foram provenientes de 5.011 municípios que aderiram ao PMAQ-AB, ciclo 2.

A Figura 1 demonstra que a TMI no Brasil apresentou redução de $45,07 \%$ entre 2000 $(24,14 / 1000$ nascidos vivos - NV) e 2015
$(13,26 / 1000 \mathrm{NV})$. A TMI média no período de 2000 a 2015 foi maior nas regiões Norte $(\mathrm{N})$, Nordeste (NE) e Centro-Oeste (CO). A maior proporção de redução foi observada na região NE (53,79\%), variando de 30,88/1000 NV em 2000, para 14,27/1000 NV em 2015; e a menor redução foi na região CO $(22,53 \%)$, variando de $17,93 / 1000 \mathrm{NV}$ em 2000, para 13,89/1000 NV em 2015. Mesmo com as altas proporções de redução, o NE continua com a segunda maior TMI em 2015, embora as diferenças entre as regiões tenham diminuído. O NE teve ainda a maior amplitude entre a maior (Alagoas-AL) e menor (Bahia-BA) TMI (Figura 1).

Em 2000, os estados com as maiores TMI eram Paraíba (41,65/1000 NV), Acre (AC) $(41,61 / 1000$ NV) e Pernambuco (37,66/1000 $\mathrm{NV}$ ); e os estados com menor taxa eram o Distrito Federal (DF) (14,40/1000 NV), Rio Grande do Sul (RS) $(15,27 / 1000$ NV) e Santa Catarina (SC) $(17,16 / 1000 \mathrm{NV})$. Em 2015, as maiores TMI foram em Roraima (RR) (23,28/1000 NV), AC $(19,89 / 1000$ NV) e Amazonas (AM) (17,82/1000 $\mathrm{NV})$; ao passo que as menores TMI permaneceram em SC (9,54/1000 NV), RS (9,6/1000 NV) e DF (10,58/1000 NV) (Tabela 1).

Ao longo desse período, houve grande variação das Unidades Federativas com maiores TMI, especialmente nas regiões N e NE. Quatro estados do N (AM, Pará (PA), RR, Rondônia (RO)), quatro do NE (AL, Piauí (PI), BA, Maranhão (MA)) e três do CO (Mato Grosso (MT), Goiás (GO), DF) apresentaram piora da TMI em 15 anos. Por outro lado, todas as unidades federativas (UF) do Sul (S) e Sudeste (SE) mantiveram ou melhoraram suas taxas a nível nacional (Tabela 1 ).

A Figura 2 apresenta o cluster diferencial sobre a MI no período 2000 a 2015. Foram considerados significantes para análise 749 municípios. Desses, 153 municípios apresentaram alta TMI, 294 apresentaram baixa TMI, 211 tiveram baixa TMI próximos a municípios com alta TMI, e 91 municípios tiveram alta TMI próximos a municípios com baixa TMI.

A extensão de área nas regiões N e NE que não apresentaram melhora na TMI é maior do que nas regiões S, SE e CO. Destaca-se áreas próximas às capitais Boa Vista e Macapá, onde não houve melhora na TMI. Destaca-se que as regiões $\mathrm{N}$ e NE tiveram grandes áreas próximas que apresentaram melhora na TMI, como em Manaus e área adjacente. Áreas nas regiões NE, SE, $\mathrm{S}$ e $\mathrm{CO}$ apontaram tendência de diminuição da TMI ao longo do período, como no município de Belo Horizonte. Observa-se ainda nessas regiões 


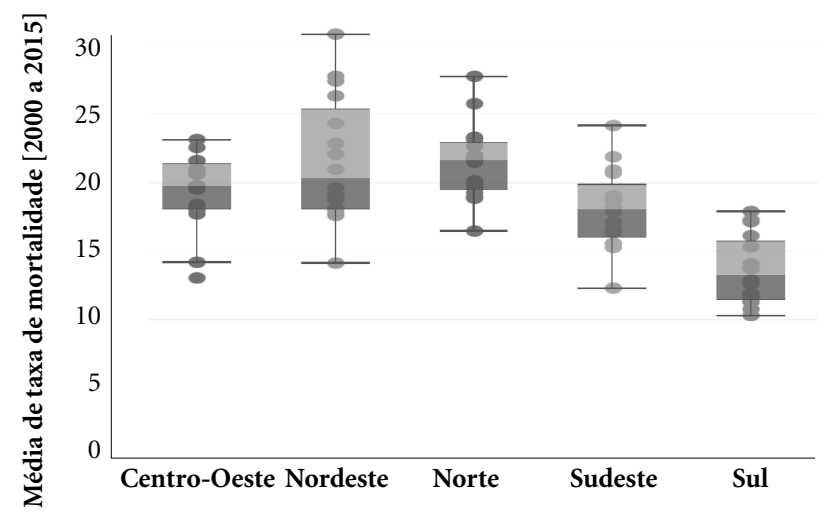

Figura 1. Média da taxa de mortalidade infantil por região brasileira. Brasil, 2000-2015.

Fonte: Elaborado pelos autores.

do país pequenas áreas próximas com altas TMI, com destaque ao estado do Mato Grosso do Sul (Figura 2).

A Tabela 2 e a Figura 3 apresentam a análise de GWR realizada com dados do PMAQ-AB, IBGE, DATASUS e artigos.

No Brasil, a TMI mostrou-se inversamente associada às variáveis acessibilidade aos serviços de alta complexidade, estrato da gestão em saúde e porte populacional, referência para o parto, taxa de nascidos vivos, renda per capita e taxa de desemprego. A variável infraestrutura das UBS demonstrou-se diretamente associada a TMI (Tabela 2).

$\mathrm{Na}$ análise por região do país, a variável acessibilidade aos serviços de alta complexidade esteve inversamente associada às regiões $\mathrm{NE}, \mathrm{N}$ e $S$ e a variável estrato da gestão em saúde e porte populacional com as regiões CO, NE e SE. A variável referência para o parto apresentou associação direta com todas as regiões brasileiras, exceto a região Norte (Tabela 2).

A taxa de nascidos vivos teve associação inversa com as regiões $\mathrm{CO}, \mathrm{NE}, \mathrm{SE}$ e $\mathrm{S}$; e a taxa de desemprego com as regiões CO, N e SE. A variável renda per capita apresentou associação inversa com todas as regiões (Tabela 2).

As áreas brancas nos mapas da Figura 3 tratam-se de municípios em que não houve a presença do PMAQ-AB ou que não foram analisados pela regressão devido ao número de missings presentes naquela região. O mapa $\mathrm{R} 2$ por quantil apresenta nas áreas de cor mais forte, regiões em que a equação para associar a variação entre os preditores e o desfecho foi mais precisa. Verificou-se que as regiões com dados mais precisos ocorreram em quase todos os estados (Figura 3 ).

O mapa resíduos (Figura 3), plotou os resíduos da regressão, em que, quanto mais claro melhor, pois a regressão ficou próxima do valor. Dessa forma, os dados demonstraram-se satisfatórios para uma análise de regressão, exceto nos estados Acre, Amazonas, Tocantins, e os da região CO.

Nos mapas seguintes, a cor verde é a área em que houve associação direta da variável com o desfecho, e a cor vermelha a área em que houve associação inversa. A maioria dos municípios das regiões do N, NE e S apresentaram a acessibilidade ao serviço de alta complexidade como um evento para redução da MI, enquanto que a maioria dos municípios das regiões SE e CO apresentaram associação direta (Mapa Acessibilidade, Figura 3).

As regiões NE, SE e CO apresentaram maioria dos municípios com associação inversa entre o desfecho e o estrato classificado pela gestão em saúde e porte populacional, fato este observado na minoria dos municípios das regiões $\mathrm{N}$ e S (Mapa Estrato, Figura 3). Todas as regiões do Brasil apresentaram maioria dos municípios com associação direta entre o desfecho MI e a infraestrutura dos serviços de $\mathrm{AB}$, ou seja, quanto melhor a infraestrutura, maior a MI (Mapa Infraestrutura, Figura 3).

Todas as regiões, exceto a região Norte, possuem maioria dos municípios com maior MI quando maior é o referenciamento do parto 


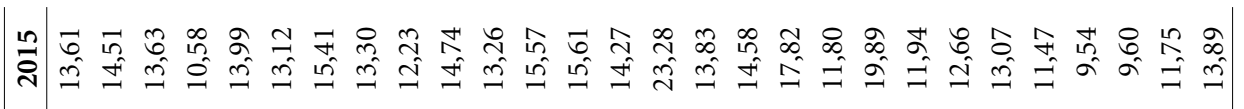

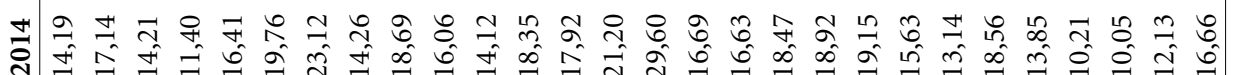

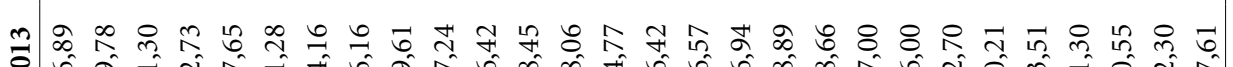

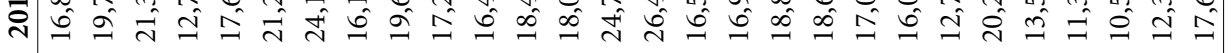

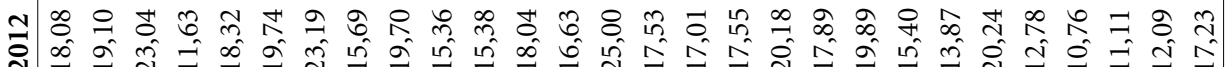

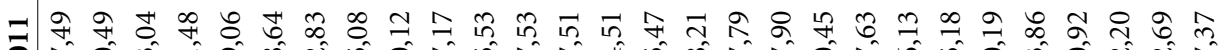

वेटी

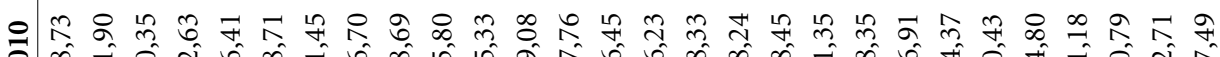

वें

영

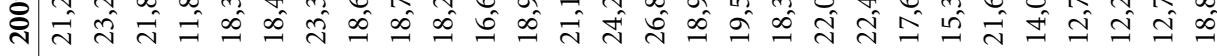

育

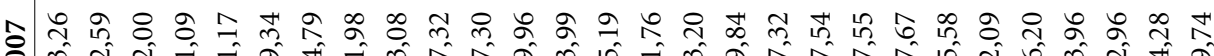

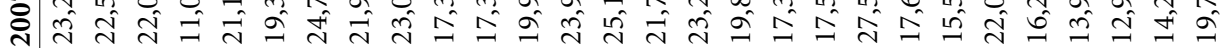

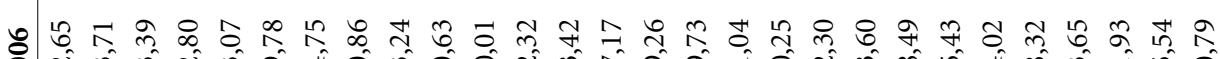

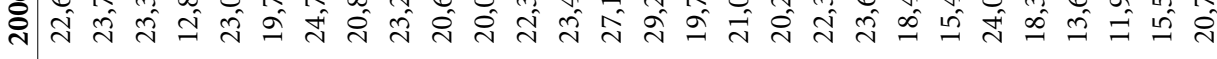

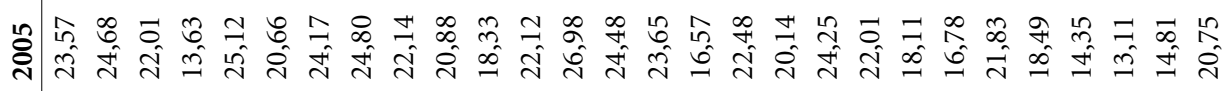

t)

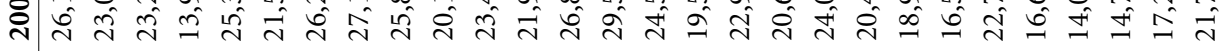

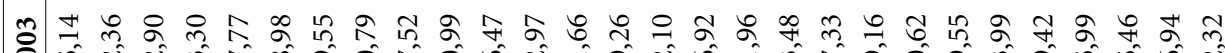

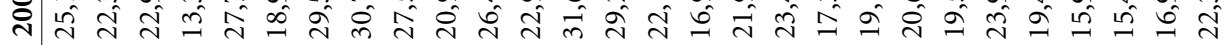

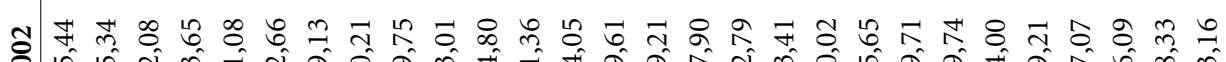

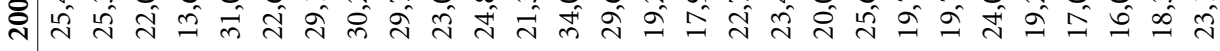

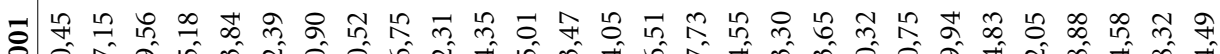

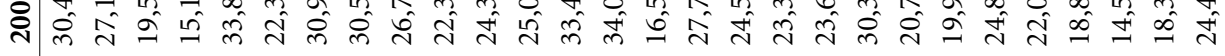

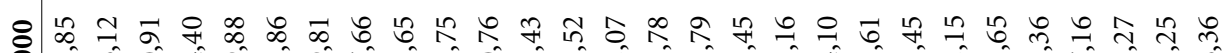

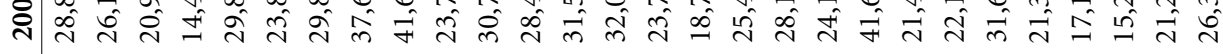

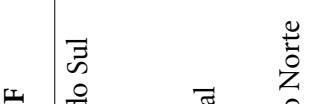

:

5
5

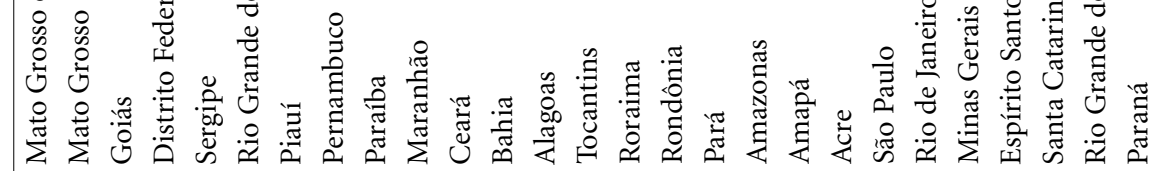

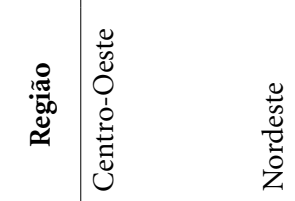

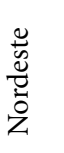

$\stackrel{0}{0}$
Zे

$\frac{\mathscr{Q}}{\mathscr{0}}$

$\bar{w}$

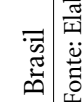




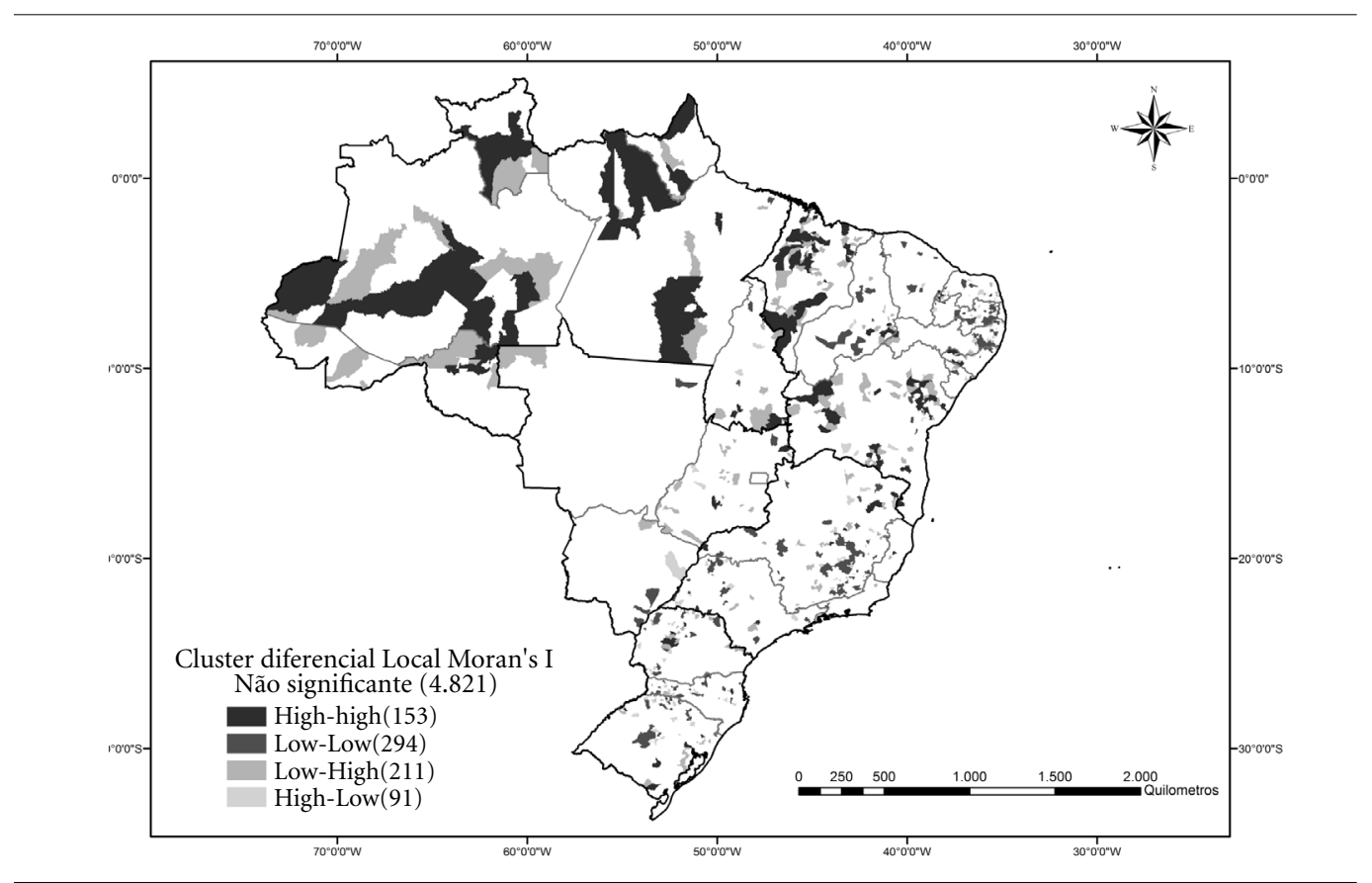

Figura 2. Cluster diferencial local Moran`s I das taxas de mortalidade infantil entre 2000 e 2015. Brasil, 20002015.

Fonte: Elaborado pelos autores.

(Mapa Referência, Figura 3). À exceção da região Sul, a maioria dos municípios das demais regiões apresentaram associação inversa entre taxa de nascidos vivos e MI, ou seja, quanto maior a taxa de nascidos vivos, menor a taxa de MI (Mapa Nascidos Vivos, Figura 3).

A maioria dos municípios de todas as regiões apontaram associação inversa da renda per capita com o desfecho (Mapa Renda, Figura 3). As regiões N, SE e CO demonstraram majoritariamente que quanto maior a taxa de desemprego menor a MI (Mapa Desemprego, Figura 3).

\section{Discussão}

Evidencia-se no país a redução da TMI, com o alcance da taxa definida pelos objetivos do milênio propostos pela Organização das Nações Unidas (ONU) até o ano 2015 27. Percebe-se o mesmo resultado quando analisada a TMI por região e por estado, entretanto, ainda considerados valores insatisfatórios e elevados se comparados à realidade de países desenvolvidos.

Para o alcance do objetivo de desenvolvimento sustentável (ODS) referente à erradicação de óbitos evitáveis nos primeiros cinco anos de vida, definido pela ONU até o ano 2030, alguns estados brasileiros, com ênfase nos estados do Norte e Nordeste, ainda necessitam de maiores investimentos para que consigam reduzir as atuais $\operatorname{taxas}^{16}$.

Apesar da crescente redução da TMI no Brasil, inclusive com a tendência de homogeneização das taxas entre as regiões brasileiras, mais avanços relacionados a aspectos socioeconômicos e demográficos e de serviços de saúde ainda são necessários. Tal fato é demonstrado com os estados que apresentaram uma redução pouco significativa quando se observa as causas desses óbitos, que poderiam ser evitadas.

Os óbitos evitáveis por ações do SUS, segundo Malta et al. ${ }^{28}$, são aqueles que se poderia reduzir por ações de imunoprevenção, na atenção prestada à mulher no pré-natal, parto e pós-parto e ao recém-nascido, na ação adequada no diagnóstico e tratamento de afecções, e com ações de promoção a saúde.

Nessa lógica, óbitos relacionados a doenças infecciosas e parasitárias, doenças do aparelho respiratório, doenças endócrinas, nutricionais e metabólicas, causas externas, algumas más formações congênitas, e afecções originadas no período perinatal, causas estas identificadas como 
Tabela 2. Análise da GWR das variáveis associadas a redução da TMI, segundo região brasileira e UF. Brasil, 2018.

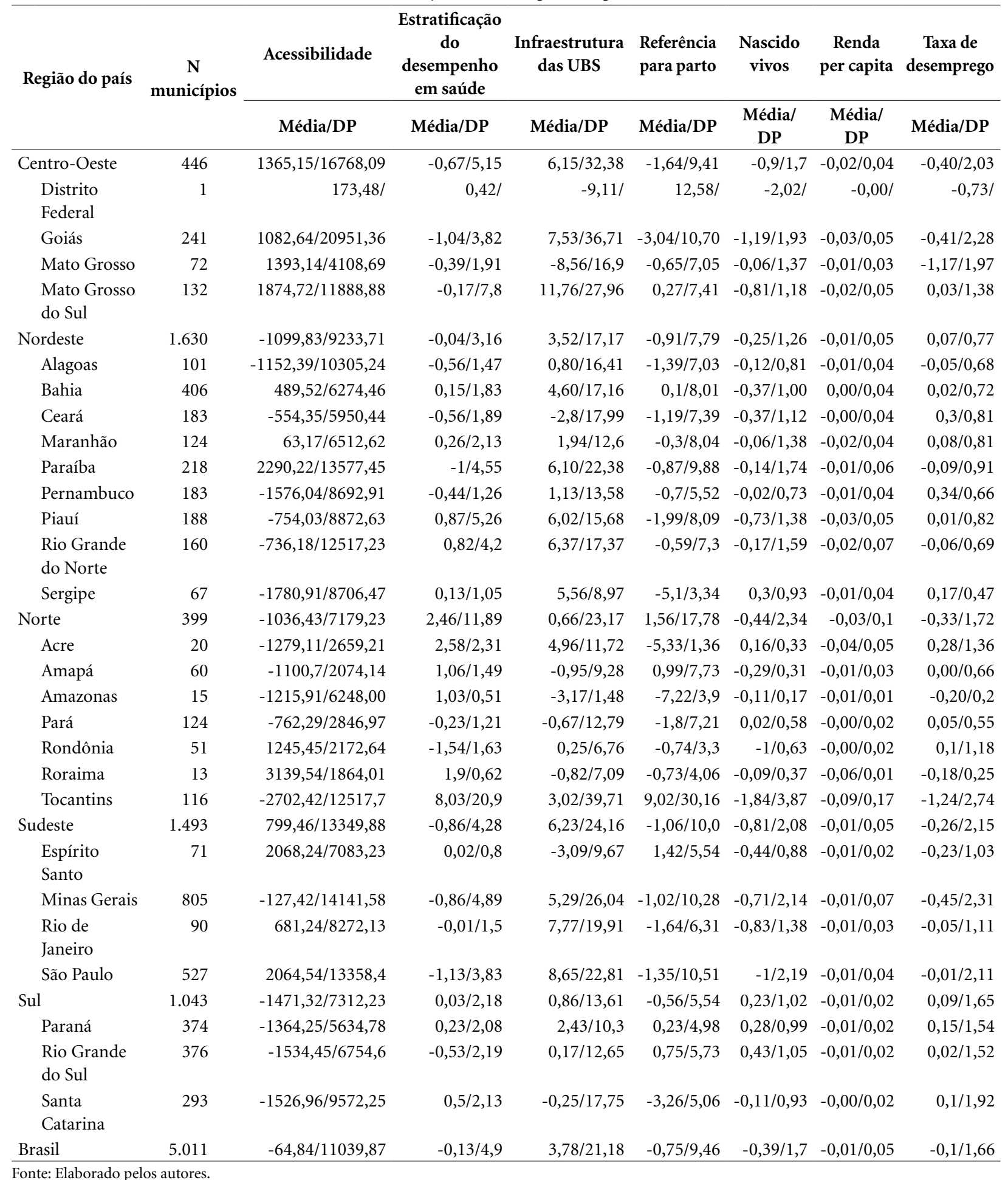

Fonte: Elaborado pelos autores. 


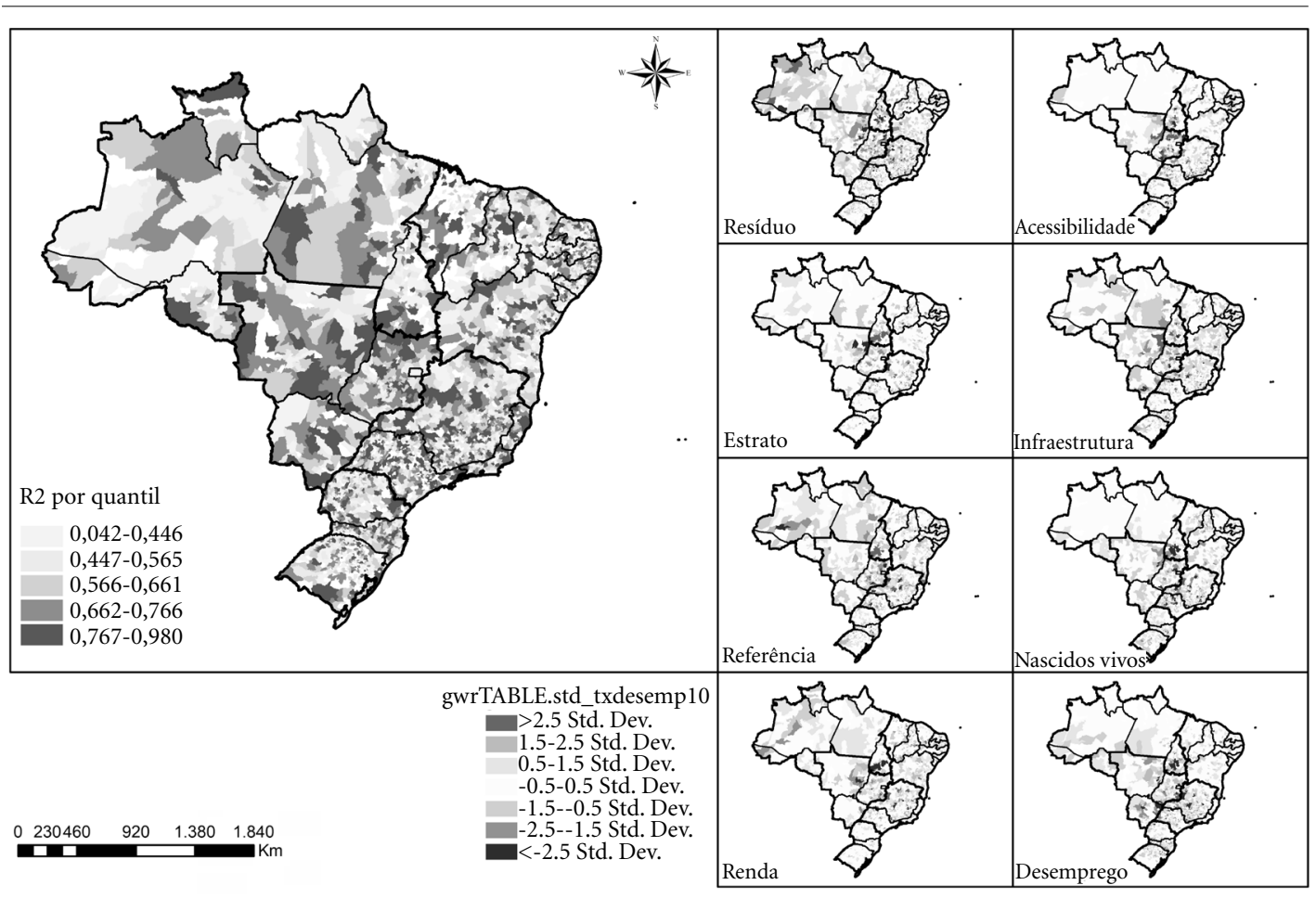

Figura 3. Mapa da GWR por município brasileiro das variáveis associadas a redução da TMI. Brasil, 2018.

Fonte: Elaborado pelos autores.

as mais prevalentes de óbito infantil, poderiam ser evitados ${ }^{28}$.

Observa-se no Brasil diferenças geográficas ainda expressivas com relação à TMI. Nos estados do Norte e Nordeste, os municípios mais distantes das capitais apresentaram menor redução nas taxas. Na região Norte, houve um contraste ainda maior que nas demais regiões do país, visto que inclusive as áreas próximas a capitais tiveram índices insatisfatórios de redução da TMI.

Victora et al. ${ }^{29}$ defendem que as desigualdades regionais diminuíram exponencialmente, com o avanço de melhoria em aspectos sociais e na expansão do acesso aos serviços de saúde. No entanto, em vistas ao progresso geral que se poderia alcançar, ainda persistem disparidades regionais em aspectos socioeconômicos, étnicos, e inclusive de serviços de saúde quando se identifica que a relação de médicos/habitantes nas regiões Norte e alguns estados do Nordeste é desproporcional à realidade dos demais estados brasileiros.

Com relação aos municípios que apresentaram melhora satisfatória na TMI mesmo que próximos a municípios com pouca redução, des- taca-se a evidência da efetividade dos avanços socioeconômicos e do serviço de saúde na prevenção de óbitos infantis. Estudos demonstram uma relação direta entre aspectos socioeconômicos e demográficos ${ }^{30-32}$ e de características do serviço de saúde ${ }^{33-35}$ com a TMI. Dentre esses pontos, destaca-se como necessário investimentos em: bolsa família no aspecto econômico, acesso dessas mulheres a educação formal, abastecimento de água e saneamento no aspecto social, estratégia saúde da família (ESF), estratégia de agentes comunitários de saúde (EACS), e acesso à atenção de saúde no aspecto serviço de saúde $\mathrm{e}^{10,29,36}$.

É fato que o acesso aos serviços de AB melhoraram, com uma alta cobertura de atendimento pré-natal, porém, quando analisada essa cobertura juntamente com a referência ao parto, percebe-se que ainda estão mal integradas ${ }^{29}$. Entretanto, este estudo aponta a importância do acesso ao serviço de alta complexidade, de uma gestão em saúde adequada nas cidades de diversos portes, e da referência ao parto para a redução da TMI.

A referência ao parto é importante para garantir à mulher a continuidade da atenção à saúde. 
Essa atividade visa ainda a integrar os serviços de saúde em seus diversos níveis de complexidade ${ }^{37}$. A organização da rede de atenção à saúde torna-se essencial nos casos em que a gestação é avaliada como de alto risco, e essa mulher precisa ser referenciada para o serviço mais próximo que atenda às suas demandas ${ }^{37}$. Grünebaum et al.$^{38}$ destacam que o parto hospitalar tem menor risco de óbito infantil que o parto domiciliar. Isso se dá pela necessidade de experiência e formação acadêmica para direcionar um parto de forma bem-sucedida.

Outro fator notado neste estudo como importante para a redução da TMI é uma maior renda per capita. No Brasil, as disparidades sociais são ainda presentes, ainda que tenham diminuído ao longo dos anos. Essas diferenças estão relacionadas diretamente com a ineficiência do governo. Dessa forma, um governo eficiente que presta serviços de qualidade e que consegue maior igualdade na distribuição de renda em seu país, pode garantir melhor condição de vida para as crianças em seus primeiros dias de vida ${ }^{39}$.

É provável que a taxa de desemprego tenha tido relação inversa com a TMI devido ser provável que mulheres desempregadas recebam auxílio governamental, como o bolsa família, as quais possuem ainda um acompanhamento próximo dos serviços de saúde, e possuem mais tempo cuidando de seus filhos por estarem em casa. Assim, o orçamento familiar não é drasticamente afetado, que seria o que levaria a uma redução com gastos para a saúde e a afetar a saúde da criança ${ }^{40}$.

A associação direta da TMI com a infraestrutura da UBS demonstra uma possível causa relacionada com o acesso e a atenção prestada pelo serviço de saúde. Melhores estruturas são mais procuradas pela população e, portanto, casos mais graves encontram-se acompanhados nessas UBS. Detollenaere et al. ${ }^{41}$ destacam que a estrutura do serviço de saúde pode ajudar ainda na redução da associação inversa encontrada entre a desigualdade de renda e a saúde de menores de um ano.
O estudo apresenta como limitação o fato de alguns valores das variáveis por município e/ ou equipe de saúde não estarem disponíveis nas fontes utilizadas, além da possível existência de sub-registros nos dados estudados. Para o discreto sub-registro de óbitos no SIM, principalmente nos estados das regiões Norte e Nordeste, caberia a utilização de modelagem para correção entre o período 2000 a 2008. Porém o próprio cálculo possui várias limitações quando utilizado por município, o que nos levou a optar pela manutenção dos dados disponíveis no SIM.

As limitações, no entanto, não inviabilizam os resultados de análise espacial de todo o território nacional e a importância destes no estudo. Destaca-se que a produção científica nacional e internacional ainda precisa ampliar pesquisas relacionadas aos serviços de saúde e à saúde da criança, com ênfase à mortalidade infantil ${ }^{12,34}$.

\section{Conclusão}

Verificou-se nesse estudo uma crescente redução da TMI entre o período de 2000 a 2015. Apesar dos crescentes investimentos para melhorias nas questões socioeconômicas e demográficas e na saúde da população brasileira, ainda se percebe diferenças significativas entre as regiões brasileiras, no que se refere ao desfecho desse estudo.

A efetivação de políticas de saúde voltadas para o foco na redução das TMI, com ênfase nos fatores que se mostraram associados, poderá fazer com que o Brasil alcance todas as metas propostas pelas Nações Unidas até o ano 2030.

O estudo mostrou a importância das pesquisas espaciais e temporais, para analisar os avanços e retrocessos vivenciados no decorrer dos anos no local de análise. O foco na relação entre a TMI e o serviço prestado pela APS proporcionou refletir a necessidade de investimentos nesse nível de atenção, visto que o mesmo se demonstrou resolutivo na redução dos óbitos infantis. 


\section{Colaboradores}

ANP Pasklan trabalhou na redação do manuscrito, análise dos dados, aprovação da versão final do artigo. RCS Queiroz trabalhou na concepção da proposta, coordenação da coleta dos dados, análise dos dados, revisão e aprovação da versão final do artigo. TAH Rocha trabalhou na concepção da proposta inicial do projeto, coordenação da coleta dos dados, análise dos dados, revisão e aprovação da versão final do artigo. NCS Rocha trabalhou na concepção da proposta inicial do projeto, coordenação da coleta dos dados, análise dos dados, revisão e aprovação da versão final do artigo. AS Tonello trabalhou na coordenação da coleta dos dados, análise dos dados. JRN Vissoci trabalhou na coordenação da coleta dos dados, análise dos dados. E Tomasi trabalhou na coordenação da coleta dos dados, análise dos dados. E Thumé trabalhou na coordenação da coleta dos dados, análise dos dados. C Staton trabalhou na coordenação da coleta dos dados, análise dos dados. EBAF Thomaz trabalhou na concepção da proposta inicial do projeto, redação do manuscrito, análise dos dados, coordenação regional da coleta dos dados, revisão e aprovação da versão final do artigo.

\section{Referências}

1. World Health Organization (WHO). Declaration of Alma Ata. Health for All by 2000. In: International Conference on Primary Health Care. Kazakhstan: WHO; 1978.

2. Puras D. Universal Health Coverage: A Return to Alma -Ata and Ottawa. Health Hum Rights 2016; 18(2):7-10.

3. André SCS, Takayanagui AMM. Atenção Primária à Saúde como instrumento para o alcance dos Objetivos de Desenvolvimento do Milênio. Rev APS 2017; 20(1):130-139.

4. Gaiva MAM, Fujimori E, Sato APS. Fatores de risco maternos e infantis associados à mortalidade neonatal. Texto Contexto Enferm 2016; 25(4):e2290015.

5. Flôr CR, Oliveira CDL, Cardoso CS, Rabelo CF, Gontijo BL, Carvalho S. Primary health care as assessed by health professionals: comparison of the traditional model versus the Family Health Strategy. Rev Bras Epidemiol 2017; 20(4):714-726.

6. Starfield B. Atenção primária: equilíbrio entre necessidades de saúde, serviços e tecnologia. Brasília: UNESCO; 2002.

7. Organização das Nações Unidas (ONU). The Sustainable Development Agenda [Internet]. 2015 [acessado 2020 fev 20]. Disponível em: https://sustainabledevelopment.un.org.

8. Berelie Y, Yismaw L, Tesfa E, Alene M. Risk factors for under-five mortality in Ethiopia: Evidence from the 2016 Ethiopian Demographic and Health Survey. $S$ Afr J Child Health 2019;; 13(3):137-140.

9. Varela AR, Schneider BC, Bubach S, Silveira MF, Bertoldi AD, Duarte LSM, Menezes AMB, Domingues MR, Bassani DG. Fetal, neonatal, and post-neonatal mortality in the 2015 Pelotas (Brazil) birth cohort and associated factors. Cad Saude Publica 2019; 35(7):e00072918.

10. Hlongwa M, de Wet N. Demographic and socioeconomic factors associated with under-5 mortality in KwaZulu-Natal, South Africa. S Afr J Child Health 2019; 13(4):174-179.

11. Rodrigues NCP, Monteiro DLM, Almeida AS, Barros MBL, Pereira NA, O’Dwyer G. Temporal and spatial evolution of maternal and neonatal mortality rates in Brazil, 1997-2012. J Pediatr (Rio J.) 2016; 92(6):567573.

12. Conroy N, Gilmore B. Child mortality and the Sustainable Development Goals: a challenge and an opportunity. Ir J Med Sci 2017; 186(2):357-358.

13. Sauvegrain P, Rico-Berrocal R, Zeitlin J. Why is perinatal and infant mortality high in the Seine-Saint-Denis district? A consultation with healthcare providers using a Delphi process. J Gynecol Obstet Biol Reprod (Paris) 2016; 45(8):908-917.

14. Karra M, Fink G, Canning D. Facility distance and child mortality: a multi-country study of health facility access, service utilization, and child health outcomes. Int J Epidemiol 2017; 46(3):817-826.

15. Turci M, Costa ML, Macinko J. Avaliação da Atenção Primária e a percepção do usuário sobre a qualidade da assistência: um estudo em cidade brasileira. APS Rev 2019; 1(1):3-7.

16. Macinko J, Harris MJ, Rocha MG. Brazil's national program for improving primary care access and quality. J Ambulatory Care Manage 2017; 40(2):S4-S11. 
17. Departamento de Informática do Sistema Único de Saúde (DATASUS) [Internet]. 2018 [acessado 2018 jul 12]. Disponível em: http://www2.datasus.gov.br/ DATASUS/index.php.

18. Instituto Brasileiro de Geografia e Estatística (IBGE) [Internet]. 2018 [acessado 2018 jul 12]. Disponível em: https://www.ibge.gov.br.

19. Programa de Melhoria do Acesso e da Qualidade da Atenção Básica (PMAQ-AB) [Internet]. 2018 [acessado 2018 jul 12]. Disponível em: https://aps.saude.gov. br/ape/pmaq/ciclo2/.

20. Rocha TAH, Silva NC, Amaral PV, Barbosa ACQ, Rocha JVM, Alvares V, Almeida DG, Thumé E, Thomaz EBAF, Queiroz RCS, Souza MR, Lein A, Lopes DP, Staton CA, Vissoci JRN, Facchini LA. Addressing geographic access barriers to emergency care services: a national ecologic study of hospitals in Brazil. Int $J$ Equity Health 2017; 16(1):149.

21. Calvo MCM, Lacerda JT, Colussi CF, Schneider IJC, Rocha TAH. Estratificação de municípios brasileiros para avaliação de desempenho em saúde. Epidemiol Serv Saude 2016; 25(4):767-776.

22. Anselin L. Local indicators of spatial association LISA. Georg Anal 1995; 27(2):93-115.

23. Scott LM, Janikas MV. Spatial Statistics in ArcGIS. In: Fischer M, Getis A. Handbook of Applied Spatial Analysis. Berlin: Springer; 2010.

24. Wong DWS. Geostatistics as measures of spatial segregation. Urban Geogr 1999; 20(7):635-647.

25. Fotheringham AS, Brunsdon C, Charlton M. Geographically Weighted Regression: The Analysis of Spatially Varying Relationships. Reino Unido: University of Newcastle, John Wiley \& Sons; 2003.

26. Anselin L, Syabri I, Kho Y. GeoDa: An Introduction to Spatial Data Analysis. In: Fischer M, Getis A. Handbook of Applied Spatial Analysis. Berlin: Springer; 2010.

27. Organização das Nações Unidas (ONU). United $\mathrm{Na}$ tions Millennium Declaration [Internet]. 2000 [acessado 2020 fev 20]. Disponível em: http://www.un.org/ millennium/declaration/ares552e.pdf.

28. Malta DC, Sardinha LMV, Moura L, Lansky S, Leal MC, Szwarcwald CL, França E, Almeida MF, Duarte EC, Grupo Técnico. Atualização da lista de causas de mortes evitáveis por intervenções do Sistema Único de Saúde do Brasil. Epidemiol Serv Saude 2010; 19(2):173-176.

29. Victora CG, Aquino EML, Leal MC, Monteiro CA, Barros FC, Szwarcwald CL. Saúde de mães e crianças no Brasil: progressos e desafios. Lancet 2011; 6736(11):60134-60138.

30. Silva AF, Silva JP. Mortalidade infantil evitável em Minas Gerais: perfil epidemiológico e espacial. Rev Bioet 2020; 28(2):276-280.

31. Pasternak S. Habitação e saúde. Revista USP 2016; 30(86):51-66.
32. Silva VA, Esperidião F. Saneamento básico e seus impactos na mortalidade infantil e no desenvolvimento econômico da região Nordeste. Scientia Plena 2017; 13(10):109905.

33. Rodrigues EC, Alves BCA, Veiga GL, Adami F, Carlesso JS, Figueiredo FWS, Azzalis LA, Junqueira VBC, Fonseca ALA, Fonseca FLA. Neonatal mortality in Luanda, Angola: what can be done to reduce it? J Hum Growth Dev 2019; 29(2):161-168.

34. Areco KCN, Konstantyner T, Taddei JAAC. Tendência secular da mortalidade infantil, componentes etários e evitabilidade no Estado de São Paulo - 1996 a 2012. Rev Paul Pediatr 2016; 34(3):263-270.

35. Tavares LT, Albergaria TFS, Guimarães MAP, Pedreira RBS, Pinto Junior EP. Mortalidade infantil por causas evitáveis na Bahia, 2000-2012. RECIIS 2016; 10(3):110.

36. Lima JC, Mingarelli AM, Segri NJ, Zavala AAZ, Takano OA. Estudo de base populacional sobre mortalidade infantil. Cien Saude Colet 2017; 22(3):931-939.

37. Gonçalves MF, Teixeira EMB, Silva MAS, Corsi NM, Ferrari RAP, Pelloso SM, Cardelli AAM. Pré-natal: preparo para o parto na atenção primária à saúde no sul do Brasil. Rev Gaucha Enferm 2017; 38(3):e0063.

38. Grünebaum A, McCullough LB, Arabin B, Brent RL, Levene MI, Chervenak FA. Neonatal Mortality of Planned Home Birth in the United States in Relation to Professional Certification of Birth Attendants. PLoS One 2016; 11(5):e0155721.

39. Ortega B, Sanjuán J, Casquero A. Determinants of efficiency in reducing child mortality in developing countries. The role of inequality and government effectiveness. Health Care Manag Sci 2017; 20(4):500516.

40. Tejada CAO, Triaca LM, Liermann NH, Ewerling F, Costa JC. Crises econômicas, mortalidade de crianças e o papel protetor do gasto público em saúde. Cien Saude Colet 2019; 24(12):4395-4404.

41. Detollenaere J, Desmarest AS, Boeckxstaens P, Willems $\mathrm{S}$. The link between income inequality and health in Europe, adding strength dimensions of primary care to the equation. Soc Sci Med 2018; 201:103-110.

Artigo apresentado em 02/04/2020

Aprovado em 06/11/2020

Versão final apresentada em 08/11/2020

Editores-chefes: Romeu Gomes, Antônio Augusto Moura da Silva 